\title{
Adaptive Detector of Spare Range-spread Target Based on AR Model
}

\author{
Gu Xinfeng ${ }^{1}$, Xu Zhengfeng ${ }^{1}$, Yan Shuqiang ${ }^{1}$, Hao Xiaolin ${ }^{2}$ \\ ${ }^{1}$ China Satellite Maritime Tracking \& Control Department, Jiangyin, China \\ ${ }^{2}$ Yantai Electricity and Economy Technical Institute, Yantai, China
}

\section{Email address:}

y6ckgxf@126.com (Gu Xinfeng)

\section{To cite this article:}

Gu Xinfeng, Xu Zhengfeng, Yan Shuqiang, Hao Xiaolin. Adaptive Detector of Spare Range-spread Target Based on AR Model. Science Discovery. Vol. 6, No. 3, 2018, pp. 193-198. doi: 10.11648/j.sd.20180603.19

Received: January 29, 2018; Accepted: June 21, 2018; Published: June 26, 2018

\begin{abstract}
The detecting of spare range-spread target is discussed in this paper based on the AR model of clutter. Compound-Gaussian clutter is modeled as the AR model, and a regressive approximate generalized likelihood ratio test detector based on scatter number (SN-RAGLRT-CG) is proposed. The probability false alarm of the detector is deduced. It does not need secondary range cells to estimating the parameters of clutter. The simulation results also show that the SN-RAGLRT-CG makes full use of the information scatterers and it improves the detection performance for spare scatterer targets of the detector.
\end{abstract}

Keywords: Compound-Gaussian Clutter, Spherically Invariant Random Vector, AR-Model, Generalized Likelihood Ratio Test

\section{基于 $A R$ 模型的稀疏扩展目标自适应检测器}

\author{
顾新锋 ${ }^{1}$, 徐正峰 ${ }^{1}$, 严树强 ${ }^{1}$, 郝晓琳 ${ }^{2}$ \\ ${ }^{1}$ 中国卫星海上测控部, 江阴, 中国 \\ 2 烟台电力经济技术研究所, 烟台, 中国
}

\section{邮箱}

y6ckgxf@126.com（顾新锋）

摘要：采用AR模型对杂波进行建模，结合目标的稀疏散射点特性，研究了距离扩展目标的自适应检测问题。复合高斯 杂波采用AR模型建模, 利用目标稀疏散射点的先验信息, 采用近似广义似然比检验(AGLRT)原理,结合迭代估计方法, 提出了复合高斯杂波背景下基于散射点个数的迭代近似广义似然比检测器(SN-RAGLRT-CG), 并从理论上分析得出了 检测器虚警概率表达式。仿真结果表明, SN-RAGLRT-CG充分利用了目标散射点的先验信息, 在不同检测窗口宽度和 不同 $\mathrm{N}$ 值的情况下, 其性能都要优于现有RAGLRT-CG和RAGLRT-GCC, 并且SN-RAGLRT-CG不需要辅助距离单元估 计杂波协参数就可以实现目标的自适应检测, 能够较好的实现稀疏扩展目标的自适应检测。

关键词：复合高斯杂波, 球不变随机向量, AR模型, 广义似然比检验 


\section{1. 引言}

当雷达距离分辨率较低时, 杂波可以采用高斯模型进 行建模, 对于高斯杂波背景下雷达目标的检测方法, 在文 献 $[1,2]$ 中已进行了广泛的研究。随着雷达分辨率的提到, 实测数据表明, 在低掠地角条件下观测时, 高斯杂波模型 不再适用，但可以采用复合高斯杂波模型 $[3,4]$ 。在高分 辨率的情况下, 一方面, 雷达会接收到类似于目标的尖峰, 这种含尖峰的复合高斯杂波可用球不变随机向量 SIRV(spherically invariant random vector, SIRV)进行建模 $[5,6]$; 另一方面, 目标的多个散射点回波被分离, 出现 在不同的距离单元中, 形成距离扩展目标。SIRV是由空 间上变化较慢的纹理分量和变化较快的散斑分量的乘积 得到, 其中, 纹理分量表征杂波的功率水平, 而基于中心 极限定理的局部有效性, 散斑分量为高斯随机向量[7]。在 SIRV杂波背景下的扩展目标自适应检测器通常采用两步 法来实现, 即先假设杂波协方差矩阵结构已知, 得到检测 器, 再利用被检测单元以外的辅助单元回波信号对协方差 矩阵结构进行估计得到自适应检测器 $[4,8]$ 。两步法自适 应检测器的一个共同特点是需要一定数量的辅助单元数 据, 这些数据需要满足独立同分布条件, 并且和被检测单 元具有相同的协方差矩阵结构。例如, 雷达采用 $N$ 个相干 脉冲检测目标时, 要得到满意的检测性能, 需要 $2 N$ 以上个 满足上述条件的辅助数据单元。两步法检测器的理论分析 结果表明, $N$ 越大, 检测器对微弱目标的检测能力也就越 强。在实际应用中, 为了能够有效地检测微弱目标, 需要 更多的脉冲数进行积累, 很难得到 $2 N$ 以上个满足独立同分 布的辅助数据单元, 从而造成自适应检测器的性能严重下 降 [9, 10]。

对于雷达干扰回波可以采用阶数在 $1 \sim 5$ 之间的低阶 $\mathrm{AR}$ 模型建模[11]。在点目标检测中, 文献[11]研究了基于 $\mathrm{AR}$ 模型的确知信号检测, 文献[12]利用 $\mathrm{AR}$ 模型对杂波进 行建模, 用于检测复幅度信息未知的确知信号。文献[13] 研究了点目标的多通道AR模型检测, 文献[14]进一步研究 了采用AR模型建模的杂波背景下距离扩展目标的检测, 但只从杂波的角度进行考虑, 但对于距离扩展目标, 并不 是所有距离单元都有目标反射回波, 目标回波实际上集中 在少数几个单元中, 针对这散射点具有稀疏特性的距离扩

$$
\prod_{k=1}^{K} f\left(\mathbf{z}_{k} \mid \mathrm{H}_{0}, \tau_{k}\right) \cong \frac{1}{\prod_{k=1}^{K} \tau_{k}^{(N-P)}\left(\sigma^{2} \pi\right)^{K(N-P)}} \cdot \exp \left[-\frac{1}{\sigma^{2}} \sum_{k=1}^{K} \frac{1}{\tau_{k}}\left(\mathbf{u}_{k}-\mathbf{Z}_{k} \mathbf{a}\right)^{\mathrm{H}}\left(\mathbf{u}_{k}-\mathbf{Z}_{k} \mathbf{a}\right)\right]
$$

在 $\mathrm{H}_{1}$ 假设下, $\mathbf{z}_{k}$ 的近似联合概率密度函数可以表示为

$$
\begin{aligned}
\prod_{k=1}^{K} f\left(\mathbf{z}_{k} \mid \mathrm{H}_{1}, \tau_{k}, \alpha_{k}\right) \cong & \frac{1}{\prod_{k=1}^{K} \tau_{k}^{(N-P)}\left(\sigma^{2} \pi\right)^{K(N-P)}} \cdot \exp \left[-\frac{1}{\sigma^{2}} \sum_{k \in \Theta_{K}-\Theta_{h_{0}}} \frac{1}{\tau_{k}}\left(\mathbf{u}_{k}-\mathbf{Z}_{k} \mathbf{a}\right)^{\mathrm{H}}\left(\mathbf{u}_{k}-\mathbf{Z}_{k} \mathbf{a}\right)\right] \\
& \exp \left[-\frac{1}{\sigma^{2}} \sum_{k \in \Theta_{h_{0}}} \frac{1}{\tau_{k}}\left(\mathbf{u}_{k}-\mathbf{Z}_{k} \mathbf{a}-\alpha_{k} \mathbf{q}+\alpha_{k} \mathbf{S a}\right)^{\mathrm{H}}\left(\mathbf{u}_{k}-\mathbf{Z}_{k} \mathbf{a}-\alpha_{k} \mathbf{q}+\alpha_{k} \mathbf{S a}\right)\right]
\end{aligned}
$$




\section{3. 检测器设计}

基于AGLRT原理, SN-RAGLRT-CG的检测统计量可 以表示为

$$
\lambda=\frac{\max _{\boldsymbol{\tau}, \boldsymbol{\alpha}, \mathbf{a}, \Theta_{h_{0}}} \prod_{k=1}^{K} f\left(\mathbf{z}_{k} \mid \mathrm{H}_{1}, \tau_{k}, \alpha_{k}\right)}{\max _{\tau, \boldsymbol{\alpha}} \prod_{k=1}^{K} f\left(\mathbf{z}_{k} \mid \mathrm{H}_{0}, \tau_{k}\right)}, N>P
$$

式 中 , $\boldsymbol{\alpha}=\left[\alpha_{1}, \ldots, \alpha_{K}\right], \boldsymbol{\tau}=\left[\tau_{1}, \tau_{2}, \ldots, \tau_{K}\right] ，$ $\mathbf{a}=[\mathbf{a}(1), \ldots, \mathbf{a}(P)], T$ 是根据给定虚警设置的检测门限。

由于 $\Theta_{h_{0}}$ 也是未知量, 这里先假设它是已知的, 先估 计其它参数, 然后对 $\Theta_{h_{0}}$ 进行估计, 将式(4)和式(5)代入式 (6)得

$$
\begin{aligned}
& \lambda=\frac{\max _{\Theta_{h_{0}}} \max _{\boldsymbol{\tau}, \boldsymbol{\alpha}, \mathbf{a}} \prod_{k \in \Theta_{h_{0}}} f\left(\mathbf{z}_{k} \mid \mathrm{H}_{1}, \tau_{k}, \alpha_{k}\right)}{\max _{\boldsymbol{\tau}, \boldsymbol{\alpha}} \prod_{k \in \Theta_{h_{0}}} f\left(\mathbf{z}_{k} \mid \mathrm{H}_{0}, \tau_{k}\right)}, N>P \quad \text { (7) } \\
& \hat{\mathbf{a}}_{0}^{(m+1)}=\left(\sum_{k \in \Theta_{h_{0}}} \frac{\mathbf{Z}_{k}^{\mathrm{H}} \mathbf{Z}_{k}}{\left(\mathbf{u}_{k}-\mathbf{Z}_{k} \hat{\mathbf{a}}_{0}^{(m)}\right)^{\mathrm{H}}\left(\mathbf{u}_{k}-\mathbf{Z}_{k} \hat{\mathbf{a}}_{0}^{(m)}\right)}\right)^{-1}\left(\sum_{k \in \Theta_{h_{0}}} \frac{\left.\mathbf{Z}_{k}^{\mathrm{H}} \mathbf{u}_{k}-\mathbf{Z}_{k} \hat{\mathbf{a}}_{0}^{(m)}\right)^{\mathrm{H}}\left(\mathbf{u}_{k}-\mathbf{Z}_{k} \hat{\mathbf{a}}_{0}^{(m)}\right)}{\mathbf{u}^{(m)}}\right)
\end{aligned}
$$

与RAGLRT-GCC方法类似，这里也采用迭代方法来 估计参数 $\mathbf{a}$ 。

先假设 $\mathbf{a}$ 已知, 则在 $\mathrm{H}_{0}$ 假设下, $\tau_{k}$ 的最大似然估计可 以表示为

$$
\hat{\tau}_{0, k}=\frac{\left(\mathbf{u}_{k}-\mathbf{Z}_{k} \mathbf{a}\right)^{\mathrm{H}}\left(\mathbf{u}_{k}-\mathbf{Z}_{k} \mathbf{a}\right)}{(N-P) \sigma^{2}}, k \in \Theta_{h_{0}}
$$

再假设 $\tau_{k}$ 已知, 则在 $\mathrm{H}_{0}$ 假设下, $\mathbf{a}$ 的最大似然估计可 以表示为

$$
\hat{\mathbf{a}}_{0}=\left(\sum_{k \in \Theta_{h_{0}}} \frac{\mathbf{Z}_{k}^{\mathrm{H}} \mathbf{Z}_{k}}{\tau_{k}}\right)^{-1}\left(\sum_{k \in \Theta_{h_{0}}} \frac{\mathbf{Z}_{k}^{\mathrm{H}} \mathbf{u}_{k}}{\tau_{k}}\right)
$$

将式(4-48)代入式(4-49)可得到在 $\mathrm{H}_{0}$ 假设下 $\mathbf{a}$ 的迭代

同样, 在 $H_{1}$ 假设下, 先假设 $\Theta_{h_{0}}$ 已知, $\tau_{k}$ 的最大似然估计可以表示为

$$
\hat{\tau}_{1, k}=\frac{\left(\tilde{\mathbf{u}}_{k}-\tilde{\mathbf{Z}}_{k} \mathbf{a}\right)^{\mathrm{H}}\left(\tilde{\mathbf{u}}_{k}-\tilde{\mathbf{Z}}_{k} \mathbf{a}\right)}{(N-P) \sigma^{2}}, k \in \Theta_{h_{0}}
$$

在 $\mathrm{H}_{1}$ 假设下, 则 $\mathbf{a}$ 的迭代估计过程可表示为

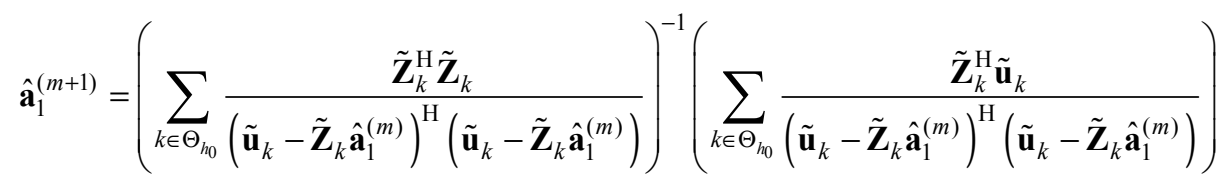

将经过 $N_{\mathrm{it}}$ 次迭代后的 $\hat{\mathbf{a}}_{0}^{\left(N_{\mathrm{it}}\right)}$ 和 $\hat{\mathbf{a}}_{1}^{\left(N_{\mathrm{it}}\right)}$ 以及 $\hat{\tau}_{0, k}$ 和 $\hat{\tau}_{1, k}$ 代入各自的似然函数中, 再将得到的似然函数代入式(7)可得

$$
\lambda=\max _{\Theta_{h_{0}}}\left[\prod_{k \in \Theta_{h_{0}}} \frac{\left(\mathbf{u}_{k}-\mathbf{Z}_{k} \hat{\mathbf{a}}_{0}^{\left(N_{\mathrm{it}}\right)}\right)^{\mathrm{H}}\left(\tilde{\mathbf{Z}}_{k} \hat{\mathbf{a}}_{1}^{\left(N_{\mathrm{it}}\right)}\right)^{\mathrm{H}}\left(\tilde{\mathbf{u}}_{k}-\tilde{\mathbf{Z}}_{k} \hat{\mathbf{a}}_{0}^{\left(N_{\mathrm{it}}\right)}\right)}{\left.\hat{\mathbf{a}}_{1}^{\left(N_{\mathrm{it}}\right)}\right)}\right]
$$

SN-RAGLRT-CG的检测统计量可以等效的表示为

$$
\lambda_{\text {SN-RAGLRT-CG }}=2(N-P-1) \max _{\Theta_{h_{0}}} \sum_{k \in \Theta_{h_{0}}} \ln \left[\frac{\left(\mathbf{u}_{k}-\mathbf{Z}_{k} \hat{\mathbf{a}}_{0}^{\left(N_{\mathrm{it}}\right)}\right)^{\mathrm{H}}\left(\mathbf{u}_{k}-\mathbf{Z}_{k} \hat{\mathbf{a}}_{0}^{\left(N_{\mathrm{it}}\right)}\right)}{\left.\left(\tilde{\mathbf{u}}_{k}-\tilde{\mathbf{Z}}_{k} \hat{\mathbf{a}}_{1}^{\left(N_{\mathrm{it}}\right)}\right)^{\mathrm{H}}\left(\tilde{\mathbf{u}}_{k}-\tilde{\mathbf{Z}}_{k} \hat{\mathbf{a}}_{1}^{\left(N_{\mathrm{it}}\right)}\right)\right]}\right.
$$


最后对 $\Theta_{h_{0}}$ 进行估计, 令

$$
l_{k}=2(N-P-1) \ln \left[\frac{\left(\mathbf{u}_{k}-\mathbf{Z}_{k} \hat{\mathbf{a}}_{0}^{\left(N_{\mathrm{it}}\right)}\right)^{\mathrm{H}}\left(\mathbf{u}_{k}-\mathbf{Z}_{k} \hat{\mathbf{a}}_{0}^{\left(N_{\mathrm{it}}\right)}\right)}{\left(\tilde{\mathbf{u}}_{k}-\tilde{\mathbf{Z}}_{k} \hat{\mathbf{a}}_{1}^{\left(N_{\mathrm{it}}\right)}\right)^{\mathrm{H}}\left(\tilde{\mathbf{u}}_{k}-\tilde{\mathbf{Z}}_{k} \hat{\mathbf{a}}_{1}^{\left(N_{\mathrm{it}}\right)}\right)}\right]
$$

式中, $\hat{\mathbf{a}}_{0}^{\left(N_{\mathrm{it}}\right)}$ 和 $\hat{\mathbf{a}}_{1}^{\left(N_{\mathrm{it}}\right)}$ 分别由式(4-50)和式(4-52)假设 $\Theta_{h_{0}}=\Theta_{K}$ 得到。对 $l_{1}, l_{2}, \ldots, l_{K}$ 从大到小排序, 得到有序序 列 $l_{i_{1}}, l_{i_{2}}, \ldots, l_{i_{K}}\left(i_{m} \in\{1,2, \ldots, K\}\right)$ 表示有序序列中第 $m$ 个元素 对应距离单元编号)。则 $\Theta_{h_{0}}$ 的估计为 $\left\{i_{1}, i_{2}, \ldots, i_{h_{0}}\right\}$, 从而 SN-RAGLRT-CG的检测统计量可以表示为

$$
\lambda_{\text {SN-RAGLRT-CG }}=\sum_{m=1}^{h_{0}} l_{i_{m}}
$$

特别地, 当 $h_{0}=K$ 时, SN-RAGLRT-CG

$$
\lambda_{\text {SN-RAGLRT-CG }}=\sum_{m=1}^{K} l_{i_{m}}=\sum_{k=1}^{K} l_{k}=\lambda_{\text {RAGLRT-CG }}
$$

这表明, 当 $h_{0}=K$ 时, SN-RAGLRT-CG 等价为 RAGLRT-CG。

\section{4. 检测统计量分析}

在有限 $N$ 值情况下 SN-RAGLRT-CG 的概率密度函数 的闭型表达式很难得到。作为近似的情况, 我们考虑在 $\mathrm{H}_{0}$ 假设下, $N \rightarrow \infty$ 时, $\lambda_{\text {SN-RAGLRT-CG }}$ 的统计特性。

当 $N \rightarrow \infty$ 时, $l_{k}$ 可以表示为

$$
\begin{aligned}
l_{k} & =2\left(N^{\prime}-1\right) \ln \left[\frac{\left(\mathbf{u}_{k}-\mathbf{Z}_{k} \mathbf{a}\right)^{\mathrm{H}}\left(\mathbf{u}_{k}-\mathbf{Z}_{k} \mathbf{a}\right)}{\left(\tilde{\mathbf{u}}_{k}-\tilde{\mathbf{Z}}_{k} \mathbf{a}\right)^{\mathrm{H}}\left(\tilde{\mathbf{u}}_{k}-\tilde{\mathbf{Z}}_{k} \mathbf{a}\right)}\right] \\
& =2\left(N^{\prime}-1\right) \ln \left[1+\frac{\left|\mathbf{y}_{k}\left(N^{\prime}\right)\right|^{2}}{\sum_{n=1}^{N^{\prime}-1}\left|\mathbf{y}_{k}(n)\right|^{2}}\right] \\
& =2\left(N^{\prime}-1\right) \ln \left(1+\frac{v_{k}}{N^{\prime}-1}\right)
\end{aligned}
$$

式中,

$$
v_{k}=\frac{\left|\mathbf{y}_{k}\left(N^{\prime}\right)\right|^{2} / 2}{\sum_{n=1}^{N^{\prime}-1}\left|\mathbf{y}_{k}(n)\right|^{2} /\left(2 N^{\prime}-2\right)}
$$

其中, $v$ 服从参数为 $\left(2,2 N^{\prime}-2\right)$ 的 $F$ 分布, 即 $v \sim F\left(2,2 N^{\prime}-2\right)$, 从而, 容易得到 $l_{k}$ 的概率密度函数为

$$
f_{l}\left(l_{k}\right)=\frac{1}{2} \mathrm{e}^{-l_{k} / 2}, l_{k} \geq 0
$$

即在 $l_{k}$ 服从均值为 2 的指数分布, 这表明, 当目标不 存在, 在 $N \rightarrow \infty$ 的极限情况下 SN-RAGLRT-CG等价为 SN-GLRT-CG, 即SN-RAGLRT-CG与SN-GLRT-CG具有相 同的虚警概率表达式。

特别地，当 $h_{0}=K$ 时， SN-RAGLRT-CG 等价为 RAGLRT-CG。由于 $\mathbf{z}_{1}, \ldots, \mathbf{z}_{K}$ 之间是相互统计独立的, 则 $l_{1}, \ldots, l_{K}$ 之间也相互统计独立, 因此, 检测统计量 $\lambda_{\text {RAGLRT-CG }} \sim \chi^{2}(2 K)$, SN-RAGLRT-CG虚警概率可表示 为

$$
P_{\mathrm{fa}}=\mathrm{e}^{-\frac{T}{2}} \sum_{m=0}^{K-1} \frac{1}{m !}\left(\frac{T}{2}\right)^{m}
$$

\section{5. 性能分析}

由于RAGLRT-CG 是 $G=K$ 时RAGLRT-GCC的特例, 也 是 $h_{0}=K$ 时 SN-RAGLRT-CG 的特例, 因此在分析 SN-RAGLRT-CG 的性能 时 与 RAGLRT-CG 及 RAGLRT-GCC进行比较。采用多主散射点 (MDS) 目标 模型对检测器的性能进行仿真分析, MDS模型见表1所示, 为了便于分析, 这里主要利用Model.4和Model.5进行分析。 在有限的 $N$ 值情况下, 很难得到SN-RAGLRT-CG检测统计 量的解析表达式, 因此, 检测门限和检测概率 $P_{\mathrm{d}}$ 都采用 Monte Carlo仿真方法得到。为了降低计算量, 虚警概率设 置为 $P_{\mathrm{fa}}=10^{-3}$, 目标信杂比定义为

$$
S C R=\frac{\varepsilon}{N \sigma_{\mathrm{c}}^{2}} \frac{\mathbf{p}^{\mathrm{H}} \boldsymbol{\Sigma}^{-1} \mathbf{p}}{\mathbf{p}^{\mathrm{H}} \mathbf{p}}=\frac{1}{N \sigma_{\mathrm{c}}^{2}} \sum_{k=1}^{K}\left|\alpha_{k}\right|^{2}\left(\mathbf{p}^{\mathrm{H}} \boldsymbol{\Sigma}^{-1} \mathbf{p}\right)
$$

式中, $\sigma_{\mathrm{c}}^{2}$ 为杂波平均功率水平。

表1 MDS目标模型。

\begin{tabular}{llllllll}
\hline 单元编号 & $\mathbf{1}$ & $\mathbf{2}$ & $\mathbf{3}$ & $\mathbf{4}$ & $\mathbf{5}$ & $\ldots$ & $\boldsymbol{K}$ \\
\hline Model.1 & $1 / K$ & $1 / K$ & $1 / K$ & $1 / K$ & $1 / K$ & $1 / K$ & $1 / K$ \\
Model.2 & $2 / 5$ & $3 / 10$ & $1 / 5$ & $1 / 10$ & 0 & 0 & 0 \\
Model.3 & $1 / 4$ & $1 / 4$ & $1 / 2$ & 0 & 0 & 0 & 0 \\
Model.4 & $3 / 4$ & $1 / 4$ & 0 & 0 & 0 & 0 & 0 \\
Model.5 & $1 / 2$ & 0 & 0 & 0 & $1 / 2$ & 0 & 0 \\
\hline
\end{tabular}

针对稀疏散射点目标模型Model.4, 图1图(a)和图1(b) 分别分析了 $N=6$ 和 $N=10$ 时，参数 $K$ 取不同值对 SN-RAGLRT-CG检测性能的影响, 并与RAGLRT-CG的检 测性能进行比较。结果表明, 对于给定的目标, SN-RAGLRT-CG对被检测窗口的长度 $K$ 的选择具有较好 的鲁棒性, 而RAGLRT-CG的性能随着 $K$ 的增加而下降。 从图1(a)可以看出, 当 $P_{\mathrm{d}}=0.8, N=6, K=10$ 时, RAGLRT-CG 
相对于 SN-RAGLRT-CG的信杂比损失约为 $3 \mathrm{~dB}$, 当 $K=25$ 时, 信杂比损失超过 $7 \mathrm{~dB}$; 从图 1 (b) 可以看出当 $N=10$ 时, $K=10$ 和 $K=25$ 对应的这一损失分别降为 $2 \mathrm{~dB}$ 和 $5 \mathrm{~dB}$ 。这表明,

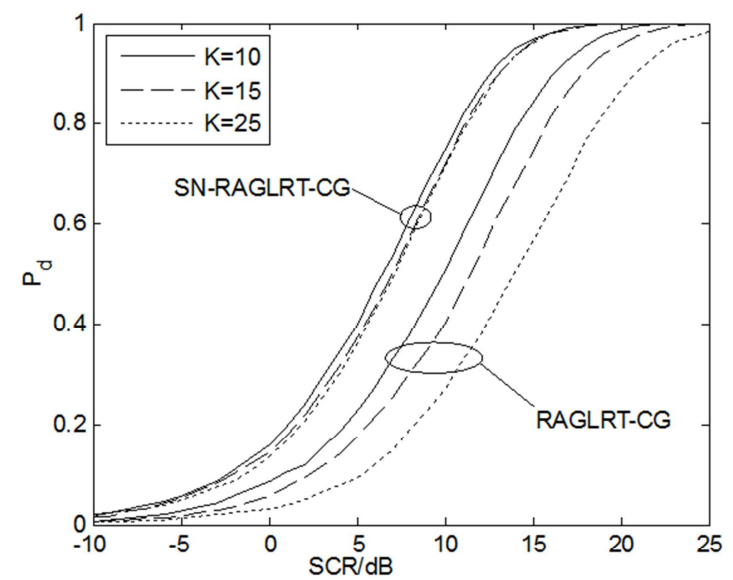

(a) $N=6$
随着 $N$ 的增加, RAGLRT-CG相对于SN-RAGLRT-CG的信 杂比损失减小。

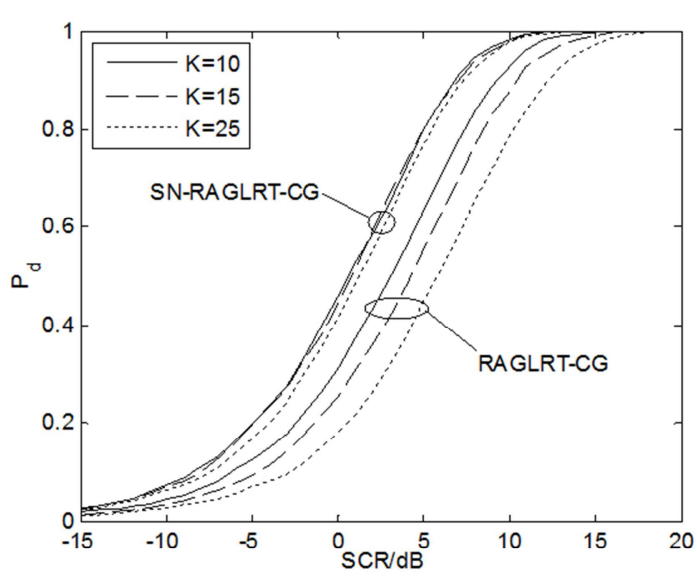

(b) $N=10$

图 $1 L=1, \gamma=0.8, N=6,10$ 时, $K=10,15,25$ 对应的SN-RAGLRT-CG和RAGLRT-CG对目标模型Model.4的检测性能曲线。

以上分析主要针对 $N$ 值较小时对 SN-RAGLRT-CG 和 RAGLRT-CG的性能的分析和比较, 并且图1的结果表明, 在稀疏散射点目标情况下，RAGLRT-CG 相对于 SN-RAGLRT-CG的信杂比损失随着 $N$ 值的增加而降低。下 面考虑 $N$ 取较大值时，在杂波分组背景下分析 SN-RAGLRT-CG、RAGLRT-GCC和RAGLRT-CG对稀疏 散射点目标的检测性能。仿真结果如图2所示。其中, 稀 疏目标模型采用Model.5, 杂波分组情况为 $\mathbf{h}=$ [5 5], 其它 参数设置为 $L=1, \gamma=0.8, K=10, N=32,64$ 。从仿真结果可以看 出, 当 $N \geq 32$ 时, RAGLRT-CG 和RAGLRT-GCC的性能曲 线几乎是重合的, 这是因为, 当 $N$ 较大时, 通过杂波分组 对AR模型参数估计精度的改善很小。当 $P_{\mathrm{d}}=0.8, N=32$ 时,

RAGLRT-CG相对于 SN-RAGLRT-CG 的信杂比损失约为 $1.5 \mathrm{~dB}$, 当 $N=64$ 时, 这一损失约为 $1 \mathrm{~dB}$, 这表明, 当杂波 分组信息或目标散射点的先验信息未知, 其 $N$ 值较大 $(N \geq 32)$ 时, 直接利用RAGLRT-CG也能得到满意的检测性能。

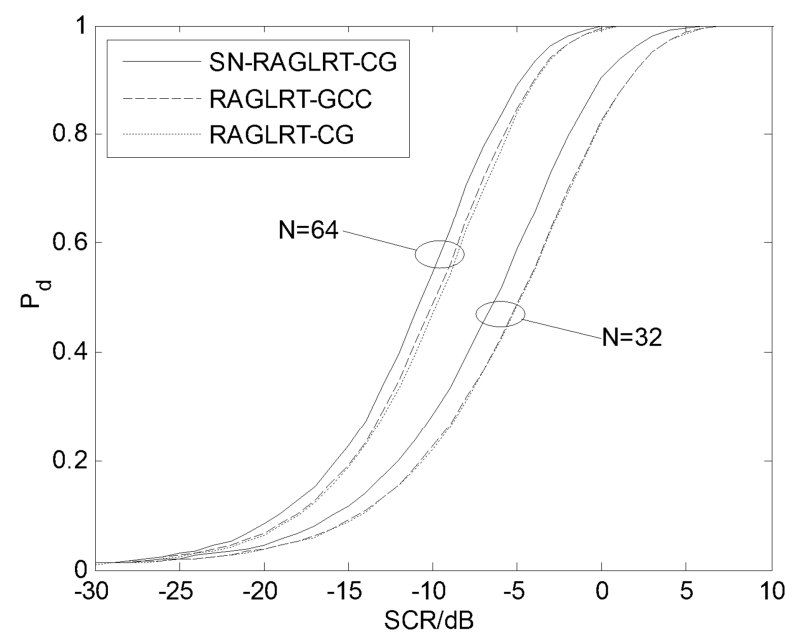

图2 $L=1, \quad \gamma=0.8, K=10, \quad \mathbf{h}=\left[\begin{array}{l}5 \\ 5\end{array}\right]$ 时, $N=32,64$ 对应的SN-RAGLRT-CG、 RAGLRT-GCC 和RAGLRT-CG 对Mode1. 5 的检测性能曲线。
从图1和图2的分析结果可以看出, SN-RAGLRT-CG 充分利用了目标散射点的先验信息, 在不同检测窗口宽度 和不同N值的情况下, 其性能都要优于现有RAGLRT-CG 和RAGLRT-GCC。

\section{6. 结束语}

本文采用AR模型对杂波进行建模, 结合目标的稀疏 散射点特性, 研究了距离扩展目标的自适应检测问题。利 用目标稀疏散射点的先验信息, 提出了 SN-RAGLRT-CG 检测器。当每个距离单元都存在目标散射点时, SN-RAGLRT-CG 等价为 RAGLRT-CG。通过对 RAGLRT-CG、RAGLRT-GCC和SN-RAGLRT-CG的性能 进行了分析比较, 仿真结果还表明, SN-RAGLRT-CG充 分利用了目标散射点的先验信息, 在不同检测窗口宽度和 不同N值的情况下, 其性能都要优于现有RAGLRT-CG和 RAGLRT-GCC, 并且SN-RAGLRT-CG不需要辅助距离单 元估计杂波协参数就可以实现目标的自适应检测, 能够较 好的实现稀疏扩展目标的自适应检测。

\section{参考文献}

[1] Robey F C, Fuhrmann D R, Kelly E J, etc. A CFAR adaptive matched filter detector[J]. IEEE Transactions on Aerospace and Electronic Systems, 1992, 28 (1): 208-216.

[2] 何友,关键,孟祥伟,等.雷达目标检测与恒虚警处理(第二 版)[M].北京,清华大学出版社,2011。

[3] 简涛,何友,苏峰,等. 高距离分辨率雷达目标检测研究现状与 进展[J].宇航学报,2010,31(12):2623-2628。 
[4] He Y, Jian T, Su F, et al. Novel range-spread target detec-tors in non-Gaussian clutter [J]. IEEETransactions on Aerospace and Electronic Systems,2010, 46: 1312-1328.

[5] 简涛,何友,苏峰,等.SIRV杂波下距离扩展目标CFAR检测器 [J].电子学报,2010,38(12):2740-2744。

[6] CONTE E, LOPS M, RICCI G. Adaptive detection schemes in compound-Gaussian clutter [J]. IEEE Transactions on Aerospace and Electronic Systems, 1998, 34(4): 1058-1069.

[7] Gerlach K. Spatially distributed target detection in non-Gaussian clutter [J]. IEEE Transactions on Aerospace and Electronic Systems, 1999, 35(3):926-934.

[8] Gu Xinfeng, Hao Xiaolin, Liu Tongling. Range-Spread Target Adaptive Detector for Non-Gaussian Clutter[J]. Science Discovery, 2017, 5(1):48-52.

[9] Melvin, W.L., Space-time adaptive radar performance in heterogeneous clutter. IEEE Transactions on Aerospace and Electronic Systems, 2000. 36(2): 621-633.
[10] McDonald K F, Blum R S. Performance characterization of space-time adaptive processing algorithms for distributed target detection in non-ideal environments[C]. Proceedings of the IEEE Radar Conference, 2002: 298-303.

[11] Alfano G, De Maio A, Farina A. Model-based adaptive detection of range-spread targets[J]. IEE Proceedings Pt. F Radar, Sonar and Navigation, 2004, 151(1): 2-10.

[12] Sheikhi A, Nayebi M M, Aref M R. Adaptive detection algorithm for radar signal in autoregressive interference $[\mathrm{J}]$. IEE Proc.-Radar Sonar Navig., 1998, 145 (5): 309-314.

[13] Haykin, S. and A. Steinhardt, Adaptive radar detection and estimation1992, New York: Wiley.

[14] Gini, F. and M. Greco, Covariance matrix estimation for CFAR detection in correlated heavy tailed clutter. Signal Processing, 2002. 82(12): 1847-1859. 